\title{
¿HACIA UN REPLANTEAMIENTO DEL CÁLCULO PROPOSICIONAL CLÁSICO?
}

\author{
EnRic Trillas, ITZiar García-HonRado \\ European Centre for Soft Computing \\ Mieres (Asturias), España \\ \{enric.trillas, itziar.garcia\}@softcomputing.es
}

\section{Resumen}

El cálculo proposicional se basa proposiciones que son tautologías, antilogías y de tipo contingente, construidas con las operaciones de disyunción, conjunción, y negación, así como con el llamado condicional material para trasladar los enunciados condicionales. Con todo ello se obtiene una estructura de álgebra de Boole, que permite equiparar el cálculo proposicional al cálculo algebraico booleano. En parte, la simplificación conceptual que se obtiene resulta de ser el orden parcial del álgebra equivalente a la afirmación del condicional material. Además, con tal metodología cabe ver qué partes del cálculo proposicional clásico pueden trasladarse, aun con limitaciones, a estructuras más débiles y, a la vez, más generales, como son los ortoretículos no booleanos o las álgebras de De Morgan.

El cálculo proposicional trata con la deducción. Por ello, el artículo lo desarrolla a partir de un operador de consecuencias en el sentido de Tarski. Ampliado el modelo deductivo, es fácil ver otras consecuencias que las usualmente consideradas en los textos elementales; en particular, se analizan cuatro esquemas de razonamiento deductivo típicos y, entre ellos, se presta especial atención a los usuales Modus Ponens y Modus Tollens.

En el caso de las estructuras algebraicas de conjuntos 'borrosos', ninguna es de álgebra de Boole ni siquiera de orto-retículo y sólo cabe trabajar, en algunos casos, con álgebras de De Morgan-Kleene. Por lo tanto el cálculo proposicional 'fuzzy' debe construirse de acuerdo con la estructura algebraica que corresponda en cada caso y una vez elegida como representación del condicional una función que verifique el Modus Ponens ('condicional borroso'). Palabras clave: Cálculo Proposicional Clásico, Álgebra de Boole, Operadores de Consecuencia Lógica, Conjeturas

Recibido: 06/06/2011. Aceptado: 19/11/2011. 


\section{Abstract}

Classical Propositional Calculus deals with propositions that are tautologies, antilogies or contingent, by means of the basic connectives disjunction, conjunction, and negation, as well as the material conditional for translating conditional statements into symbolism. Since a Boolean Algebra is obtained, Propositional Calculus could be reduced to just Boolean Calculus. This conceptual simplification comes since the partial order is equivalent to asserting the material conditional. Moreover, this methodology allows to analyze which parts of the classical calculus could be directly translated into weaker, and more general algebraic structures as, for instance, orthomodular non boolean ortholattices, and De Morgan algebras.

Propositional calculus is deductive; hence, it is developed in the paper from a Tarski's Consequence Operator. Once the deductive model is newly viewed in this way, it is easy to reach other consequences different from those usually appearing in the Textbooks. In particular, and instead of only the two well-known schemes of Modus Ponens and Modus Tollens (notwithstanding, specially considered), four similar schemes of reasoning are studied.

Among the algebraic structures of fuzzy sets, there are neither Boolean algebras, nor proper ortholattices, but only some De Morgan-Kleene algebras. So, Fuzzy Propositional Calculus should only be done in the particular algebraic structure corresponding at each case, and once it is choosen a conditional function verifying Modus Ponens (fuzzy conditional).

Keywords: Classical Propositional Calculus, Boolean Algebras, Consequence Operators, Conjectures

\section{Introducción}

Los más conocidos manuales (españoles) de Lógica Formal del siglo pasado (vid. por ejemplo, [10] [6], y [11]), solían obviar el fácil cálculo booleano que data de 1847 [4], y mostraban las pruebas de las tautologías bien por pasos, en cada uno de los cuales se aplica una de las llamadas reglas de 'inferencia', bien por medio del aún más tedioso método consistente en calcular la tabla de verdad de la presunta tautología. Ello, además, cuando la validez de las fórmulas consideradas no puede darse en otro contexto que el de las álgebras de Boole. Denotando la conjunción por ., la disyunción por + , y la negación por ', compárese, por ejemplo, cualquier prueba del Modus Tollens,

$$
(p \rightarrow q) \& q^{\prime} \rightarrow p^{\prime},
$$

en los manuales que la muestran, con la brevedad del cálculo booleano,

$$
q^{\prime} \cdot(p \rightarrow q)=q \cdot\left(p^{\prime}+q\right)=q^{\prime} \cdot p^{\prime} \leq p^{\prime},
$$

equivalente a $q^{\prime} .(p \rightarrow q) \rightarrow p^{\prime}=1$, es decir a que $(p \rightarrow q) \& q^{\prime} \rightarrow p^{\prime}$ es una tautología. Para probar que la fórmula $(p+q) \cdot(p+r) \rightarrow(p+q \cdot r)$ es una ley lógica, se requiere aplicar sucesivamente no menos de diez pasos 'inferenciales' clásicos, en tanto que con el cálculo booleano la prueba se obtiene en sólo los tres pasos algebraicos $(p+q) \cdot(p+r) \rightarrow(p+q \cdot r)=p^{\prime} \cdot q^{\prime}+p^{\prime} \cdot r^{\prime}+p+q \cdot r=$ $p^{\prime} \cdot(q \cdot r)^{\prime}+p+q \cdot r=p+(q: r)^{\prime}+q \cdot r=1$. 
El cálculo booleano permite mostrar, también rápidamente, si una fórmula lógica no es una tautología; por ejemplo, la fórmula $(p \rightarrow q) \cdot q \rightarrow p$, no es válida para cualesquiera $p$ y $q$, ya que $(q \cdot(p \rightarrow q))^{\prime}+p=q^{\prime}+\left(p^{\prime}+q\right)^{\prime}+p$ $=q^{\prime}+p \cdot q^{\prime}+p=q^{\prime}+p=1$, equivale a $q \rightarrow p=1$, o $q \leq p$, que no será siempre el caso. No es, por tanto, una tautología o ley lógica, y además quedan determinados los casos $(q \leq p)$ en los que la fórmula es válida.

No sólo los cálculos booleanos son estructurales y más simples, sino que con ellos cabe obtener otras consecuencias lógicas además de aquellas constituídas sea por los términos que aparecen en las fórmulas, sea por sus negaciones. Por ejemplo, en el caso del Modus Tollens, no sólo es $p^{\prime}$ una consecuencia de las premisas $q^{\prime}$ y $p \rightarrow q$, sino que también lo es cualquier $x$ tal que $x \geq q^{\prime} \cdot p^{\prime}$, como es el caso de $x=q^{\prime}+p^{\prime}=(p . q)^{\prime}$. Nótese que de existir algún $x$ tal que $q^{\prime} \cdot p^{\prime} \leq x \leq p^{\prime}$, también se deduce de aquellas premisas, y es una consecuencia lógica de ellas. Con frecuencia, en el razonamiento ordinario, son más interesantes tales $x$ que la consecuencia clásica $p^{\prime}$, que es la negación de uno de los datos de partida.

Además, y gracias al concepto de operador de consecuencias, o sistema deductivo, introducido por Alfred Tarski en los años treinta del siglo pasado [20], no cabe confundir 'deducción' con 'inferencia', así como hacer ver que la validez de las fórmulas depende del particular sistema deductivo que se considere. El sistema deductivo 'oculto' en aquellos manuales, corresponde al mayor de los operadores de consecuencias que cabe considerar en las álgebras de Boole; algo análogo a lo que sucede con el condicional material $a \rightarrow b=a^{\prime}+b$, que es el mayor condicional posible en las álgebras de Boole y sólo en ellas.

La nueva teoría de los llamados modelos CHE (por consecuencias, hipótesis, especulaciones, [9], [20]) permite ir aún más lejos de la deducción, que no se da en más allá del $25 \%$ de los razonamientos ordinarios [17]. Por ello, y en el reducido ámbito teórico de las álgebras de Boole, cuya abundancia de leyes limita su utilidad como marco de representación a una pequeña parte del razonamiento ordinario, este artículo presenta una aplicación de esos modelos a los cuatro casos elementales en los que las premisas son

1. $p \rightarrow q$, $p$ (Modus Ponens)

2. $p \rightarrow q, q$

3. $p \rightarrow q, p^{\prime}$

4. $p \rightarrow q$, $q^{\prime}$ (Modus Tollens),

de los cuáles los 2 y 3 no suelen considerarse en los manuales usuales. Ello tal vez, por cuanto con los métodos allí empleados sólo cabe deducir las segundas premisas, $q \circ p^{\prime}$, respectivamente. 
El razonamiento ordinario intenta, usualmente, ir más allá de las premisas disponibles y, en este sentido, aquellos manuales se quedan cortos. Por lo que respecta al estudio del razonamiento ordinario, hasta cierto punto y aún siendo algunos de ellos unos buenos textos, actualmente y por lo menos cabe considerarlos incompletos. Por ejemplo, ninguno de ellos presta la menor atención al importante razonamiento probabilístco, como hace por ejemplo el bien conocido texto [14] de 1981, y ya había hecho George Boole en su 'Laws of Thought' [5] de 1854. Lo que sigue sólo pretende ilustrar, con ejemplos elementales, una posible via para actualizar una parte de los contenidos de los cursos en los que tales manuales se hayan venido recomendando.

Téngase en cuenta que al hurtar la centralidad de la estructura de álgebra de Boole en el cálculo proposicional clásico, también se hurta la práctica del cálculo booleano que no sólo es de gran importancia para las aplicaciones, sino más sencillo que el cálculo aritmético. Por descontado que hubo alguna excepción, como es el caso de [15] que, no sólo fue mucho menos conocido que los [6] y [11], sino que no es suficientemente claro al respecto.

\section{Conceptos básicos}

A lo largo de este artículo se trabajará fundamentalmente con álgebras de Boole $\left(L, \leq,+, .{ }^{\prime} ; 0,1\right)$, estructuras en las que $(L, \leq)$ es un conjunto parcialmente ordenado con mínimo (0) y máximo (1), en el que de definen operaciones de unión (+), intersección (.) y negación ('), verificando las propiedades de idempotencia, conmutatividad, asociatividad, distributividad y leyes de De Morgan, además de los principios de no contradicción y tercero excluido y siendo la negación involutiva con la propiedad de reversibilidad respecto al orden parcial.

Así mismo se hace alusión a estructuras algebraicas más débiles que las álgebras de Boole, como los orto-retículos, en los que las únicas leyes booleanas que no se verifican son las dos distributivas, y los retículos ortomo$d$ ulares que son orto-retículos en los que es $a \leq b \Leftrightarrow b=a+a^{\prime}$. $b$. Las álgebras de De Morgan tienen todas las propiedades booleanas excepto las leyes de no-contradicción $\left(a \cdot a^{\prime}=0\right)$ y tercero-excluído $\left(a+a^{\prime}=1\right)$.

Sólo en las álgebras de Boole son indistinguibles los conceptos de incompatibilidad $(a, b=0)$ y contradicción $\left(a \leq b^{\prime}\right)$; tal indistinguibilidad no se mantiene en las otras estructuras citadas ni, mucho menos, en el razonamiento ordinario. 


\section{1.}

Dados un conjunto $\mathrm{X}$ y una familia de sus subconjuntos $\mathfrak{F} \subset P(X)$, una aplicación $\mathrm{C}: \mathfrak{F} \rightarrow \mathfrak{F}$ es un $\mathfrak{I}$-operador de consecuencias en el sentido de Tarski [20], si verifica

1. $P \subset C(P)(C$ es extensivo $)$

2. $P \subset Q \Rightarrow C(P) \subset C(Q)$ (C es monótono)

3. $C(C(P))=C^{2}(P)=C(P)$ (C es una clausura), para cualquier $P, Q$ en $\mathfrak{F}$. Un tal operador se llama

- Consistente, si $q \in C(P) \Rightarrow q^{\prime} \notin C(P)$, para todo $P \in \mathfrak{F}$, con $q^{\prime}=$ no $q$

- Compacto, si para todo $P \in \mathfrak{s}$ existe $\left\{p_{1}, \ldots, p_{n}\right\} \in \mathfrak{F}$ tal que $C(P)=$ $C\left(\left\{p_{1}, \ldots, p_{n}\right\}\right)$.

Se dice que la terna $(\mathrm{X}, \mathfrak{F}, \mathrm{C})$ es un sistema deductivo o estructura de consecuencias lógicas. Los axiomas 1,2 y 3 , pueden comprimirse en el único axioma ' $P \subset C(Q) \Leftrightarrow C(P) \subset C(Q)$ '.

Ejemplo 2.1 Sea $\mathbb{E}=\left(L ; \leq, .,+,{ }^{\prime} ; 0,1\right)$ un algebra de Boole completa y la familia

$$
\mathfrak{F}_{1}=\{P \in P \subset L ; \operatorname{Inf} P=p \wedge \neq 0\} .
$$

De considerar conjuntos $P$ de 'premisas' que sean finitos, si es $P=\left\{p_{1}, \ldots\right.$, $p_{n}$ \} es $p_{\wedge}=p_{1} \ldots p_{n}$, y al ser $0 \neq p_{\wedge} \leq p_{i}$ para todo $p_{i} \in P$, es $p i \neq 0$.

Los operadores de_nidos por

- $C^{\wedge}(P)=\left\{q \in L ; p^{\wedge} \leq q\right\}$, para todo $P \in \mathfrak{F}_{1}$

- $C \leq(P)=\{q \in L ; \exists p \in P: p \leq q\}$, para todo $P \in \mathfrak{F}_{1}$ facilitan aplicaciones $C \wedge: \mathfrak{F}_{1} \rightarrow \mathfrak{F}_{1}, y C \leq: \mathfrak{F}_{1} \rightarrow \mathfrak{F}_{1}$, que son operadores de consecuencias en la familia $\mathfrak{F}_{1}$, y verifican $C_{s}(P) \subset C_{\lambda}(P)$, para todo $P \in \mathfrak{F}_{1}$

Nótese que $C_{\lambda}(P)=C_{\lambda}\left(\left\{p_{\lambda}\right\}\right)$, muestra que $C_{\lambda}$ es compacto; además, si fuese $p_{\wedge} \leq q$ y $p_{\wedge} \leq q^{\prime}$ sería $p_{\wedge} \cdot p_{\wedge}=p_{\wedge} \leq q \cdot q^{\prime}=0$, o $p_{\wedge}=0$, que es absurdo. Por tanto, $C_{\wedge}$ también es consistente. Sin embargo y en general, $C_{s}$ no es compacto, aunque es consistente ya que de $\operatorname{ser} p_{1} \leq q$ y $p_{2} \leq q^{\prime}$, serían $p_{1} \cdot p_{2}$ $=0$, que es absurdo ya que implicaría $p_{\wedge}=0$.

Nota 2.2 En cualquier álgebra de Boole, si C es un $\mathfrak{F}_{1}$-operador de consecuencias, es $C(P) \subset C_{\lambda}(P)$ para todo $P \in F_{1}$. Es decir, $C_{\lambda}$ es el mayor de los $\mathfrak{F}_{1}$-operadores de consecuencias. Este resultado [1], sólo es válido en las álgebras de Boole.

\section{2.}

Dados un álgebra de Boole $\mathfrak{R}$, y un sistema deductivo $(L, \mathfrak{F}, C)$ con $C$ consistente,

$$
\operatorname{Conj}_{C}(P)=\left\{q \in L ; q^{\prime} \notin C(P)\right\},
$$

es el conjunto de las C-conjeturas de $P$ [19], que verifica: 
- $C(P) \subset \operatorname{Conj}_{C}(P)$, las consecuencias son conjeturas de un tipo especial.

- $P \subset \operatorname{Conj}_{C}(P)$, Conj $_{C}$ es extensivo.

- $P \subset Q \Rightarrow \operatorname{Conj}_{C}(Q) \subset \operatorname{Conj}_{C}(P), \operatorname{Conj}_{C}$ es anti-monótono.

- En general, $\operatorname{Conj}_{C}(P) \notin \mathfrak{F}$, es decir, $\operatorname{Conj}_{C}: \mathfrak{F} \rightarrow L$.

Así,

$\operatorname{Conj}_{C_{\wedge}}(P)=\left\{x \in L ; p_{\wedge} \ddagger x^{\prime}\right\} ; \operatorname{Conj}_{C s}(P)=\left\{x \in L ; \forall p \in P: p \supsetneqq x^{\prime}\right\}$, son $\mathfrak{F}_{1}$-operadores de conjeturas, respectivamente, y de $C_{s}(P) \subset C_{\Lambda}(P)$ sigue $\operatorname{Conj}_{\mathrm{C}_{\Lambda}}(P) \subset \operatorname{Conj}_{\mathrm{C}_{s}}(P)$, si $P \in \mathfrak{F}_{1}$.

\section{3.}

Los conjuntos:

- $\operatorname{Hyp}_{C}(P)=\left\{b \in \operatorname{Conj}_{C}(P) ; h^{\prime} \notin C(P) \& P \subset C(\{b\}) ; s i\{b\} \in \mathfrak{F}\right\}$, es el de las hipótesis de $P$.

- $S p_{C}(P)=\operatorname{Conj}_{C}(P)-\left[C(P) \cup H y p_{C}(P)\right]$, es el de las especulaciones de $P$.

Nótese que $\operatorname{Conj}_{C}(P)=C(P) \cup H y p_{C}(P) \cup S p_{C}(P)$ es una partición. El conjunto, $\operatorname{Ref}_{C}(P)=\operatorname{Conj}_{C}(P)^{c}=\left\{x \in L ; x^{\prime} \in C(P)\right\}$, es el de las refutaciones de $P$, con el que se obtiene la partición de $L, L=\operatorname{Conj}_{C}(P) \cup \operatorname{Ref}_{C}(P)=C(P)$ $\cup H y p_{C}(P) \cup S p_{C}(P) \cup \operatorname{Ref}_{C}(P)$.

Ejemplos 2.3 1. Con $C=C_{\wedge}$, es:

- $\operatorname{Conj}_{\wedge}(P)=\left\{x \in L ; p_{\wedge} ¥ x^{\prime}\right\}, \operatorname{Ref}_{\wedge}(P)=\left\{x \in L ; p_{\wedge} \leq x^{\prime}\right\}$

- $\operatorname{Hyp}_{{ }_{\lambda}}(P)=\left\{b \in L ; 0<b<p_{\hat{\lambda}}\right\}$

- $S p_{\wedge}(P)=\left\{s \in L ; p_{\wedge} \ddagger s^{\prime} \& p_{\wedge} N C s\right\}$, indicando con $N C=$ 'no comparable con', bajo el orden $\leq$

2. Con $C=C_{s}$, es:

- $\operatorname{Conj}_{s}(P)=\left\{x \in L ; \forall p \in P: p \sharp x^{\prime}\right\}, \operatorname{Ref}_{s}(P)=\left\{x \in L ; \exists p \in P: p \leq x^{\prime}\right\}$

- $\operatorname{Hyp}_{s}(P)=\{b \in L ; 0<b<p ; \forall p \in P\}$

- $S p_{s}(P)=\left\{s \in L ; s \in \operatorname{Conj}_{s}(P) \& s \notin C_{s}(P) \& s \notin H y p_{s}(P)\right\}$.

\section{4.}

El cálculo proposicional clásico se plantea siempre con la interpretación $a \rightarrow b=a^{\prime}+\mathrm{b}$ (condicional material) de los enunciados condicionales ' $\mathrm{Si}$ a, entonces b'. Este condicional permite asegurar el Modus Ponens y el Modus Tollens en las álgebras de Boole, ya que

$$
a \cdot(a \rightarrow b)=a \cdot\left(a^{\prime}+b\right)=a \cdot b \leq b ; b^{\prime} \cdot(a \rightarrow b)=b^{\prime} \cdot\left(a^{\prime}+b\right)=a^{\prime} \cdot b^{\prime} \leq a^{\prime},
$$

pero la verificación de estas desigualdades tanto en orto-retículos, como en álgebras de De Morgan [3], fuerza a que valgan todas las leyes del álgebra de Boole [21]: El condicional material es típicamente booleano y, además, no sirve siempre para representar todos los condicionales que aparecen en 
el lenguaje natural; por ejemplo, en el caso de condicional [7] 'Si llueve, Elisa lleva sombrero', en el que Elisa puede llevar sombrero aún sin llover, parece más razonable una de las dos interpretaciones $a^{\prime}+a \cdot b$, o $a \cdot b$, que hacen suponer que su contexto no es booleano.

Realmente, en las álgebras de Boole hay más condicionales, es decir, operaciones $\rightarrow$ verificando la desigualdad $a \cdot(a \rightarrow b) \leq \mathrm{b}$, ya que

$$
a \cdot z \leq b \Leftrightarrow z \leq a^{\prime}+b
$$

implica que los condicionales vienen caracterizados por $a \rightarrow b \leq a^{\prime}+b$, lo que muestra que el 'material' es el mayor de todos los condicionales posibles. La equivalencia anterior es característica de las álgebras de Boole, y se prueba como sigue:

1. $z \leq a^{\prime}+b \Rightarrow a \cdot z \leq a \cdot\left(a^{\prime}+b\right)=a \cdot b \leq b$.

2. $a \cdot z \leq b \Rightarrow a^{\prime}+a \cdot z=a^{\prime}+z \leq a^{\prime}+b \Rightarrow z \leq a^{\prime}+z \leq a^{\prime}+b$.

Obsérvese que $a \rightarrow b=b, a \rightarrow b=a^{\prime}, a \rightarrow b= \begin{cases}a^{\prime}+b ; & \text { si } a . b=0 \\ a \cdot b ; & \text { si } a . b \neq 0,\end{cases}$ $a \rightarrow b=a \cdot b+a^{\prime} \cdot b^{\prime}, \ldots$, verifican $a \rightarrow b \leq a^{\prime}+b$ y, por tanto, son condicionales. Nótese que de esos condicionales, sólo el material y el $a \cdot b+a^{\prime} \cdot b^{\prime}$ $=\left(a^{\prime}+b\right) \cdot\left(a+b^{\prime}\right)$, llamado bicondicional, son contrasimétricos, es decir, verifican $a \rightarrow b=b^{\prime} \rightarrow a^{\prime}$; basta que verifiquen el Modus Ponens para que también verifiquen el Modus Tollens, ya que $a \cdot(a \rightarrow b) \leq b \Rightarrow b^{\prime} \cdot\left(b^{\prime} \rightarrow\right.$ $\left.a^{\prime}\right)=b^{\prime} \cdot(a \rightarrow b) \leq a^{\prime}$. Los que no son contrasimétricos son adecuados para aquellos casos en los que no hay equivalencia entre 'Si a, entonces b' y 'Si no b, entonces no a'. Es el caso del ejemplo anterior, que, obviamente, no está claro que equivalga a 'Si Elisa no lleva sombrero, no llueve'.

¿Qué sucede con el condicional y los operadores de consecuencias $C_{\wedge}$ y $C_{s}$ ? En general, no se mantiene la validez de los mismos esquemas. Por ejemplo, con el condicional conjuntivo $a \rightarrow b=a \cdot b$, no es $a^{\prime} \in C_{s}(\{a \rightarrow b$, $\left.\left.b^{\prime}\right\}\right)=C_{s}\left(\left\{a \cdot b, b^{\prime}\right\}\right)$ puesto que $b^{\prime} \leq a^{\prime} \Leftrightarrow a \leq b$, o $a \cdot b \leq a^{\prime} \Rightarrow a \cdot b=0$, no valen siempre.

\section{Notas 2.4}

- En [21] se ha probado que, tanto en los orto-retículos, como en las álgebras de Boole, la desigualdad $a \cdot\left(a^{\prime}+b\right) \leq b$ fuerza que el retículo sea un álgebra de Boole. Por ello, que el condicional material verifque el Modus Ponens, es decir, sirva para deducir 'hacia adelante', es una propiedad exclusivamente booleana, como también lo es que verifique el Modus Tollens, que sirva para deducir 'hacia atrás'.

- Para ilustrar la no universalidad del condicional material en el razonamiento ordinario, consideremos el enunciado condicional introducido por Bertrand Russell [16], 
Si los cerdos tienen alas, hay animales con alas cuya carne es sabrosa $=a \rightarrow b$, que es difícilmente aceptable como 'verdadero'. De interpretar $a \rightarrow$ $b$ como $a^{\prime}+b$, al ser a 'falso' $y$, por tanto, $a$ ' 'verdadero', $a \rightarrow b$ sería 'verdadero'. Sin embargo, de interpretar $a \rightarrow b$ como $a \cdot b$, resultaría que $a \rightarrow b$ es 'falso'. Con la primera representación, el enunciado de Russell debe ser contrasimétrico, es decir, equivalente a

Si no hay animales con alas cuya carne es sabrosa, los cerdos no tienen alas,

un enunciado tal vez menos 'sorprendente' que el de Russell. Con la segunda interpretación no sólo no es contrasimétrico, sino que $b^{\prime} \rightarrow$ $a^{\prime}=b^{\prime} \cdot a^{\prime}=0.1=0$, con lo que el contrasimétrico también es 'falso'.

- Veamos que, por ejemplo, el condicional material no puede emplearse para deducir 'hacia adelante' en las álgebras de De Morgan. Si en tales álgebras se verificase el Modus Ponens, es decir, la inecuación ala $\rightarrow b) \leq b$, con $a \rightarrow b=a^{\prime}+b$, de $a \cdot\left(a^{\prime}+b\right)=a \cdot a^{\prime}+a \cdot b \leq b$, ya que en ellas vale la ley distributiva, con $b=0$ se seguiría $a \cdot a^{\prime}=0$, una ley que no se verifica en las álgebras de De Morgan. Nótese que de verificarse, para todo a del álgebra valdría $a \cdot a^{\prime}=0$, es decir, se trataría de un álgebra de Boole. Con mayores complicaciones técnicas (vid. [21]) puede probarse, como se ha dicho anteriormente, que lo mismo sucede en el caso de los orto-retículos.

- Nota sobre la interpretación del operador condicional.

Al representar un enunciado lingüístico condicional 'Si a, entonces b' $o$ ' $b$, si a', por una operación condicional $\rightarrow$ y para hacer un razonamiento deductivo hacia adelante, no sólo debe verificarse la inecuación del Modus Ponens $a \cdot(a \rightarrow b) \leq b$, sino que $\rightarrow$ ha de 'interpretar' correctamente el significado del enunciado, es decir, lo que se quiere decir con él.

Por ejemplo, dado el enunciado condicional 'Si llueve, entonces llevo paraguas', cualquiera de los condicionales $a \rightarrow b=a^{\prime}+a \cdot b$ (no llueve o llueve y llevo paraguas), o $a \rightarrow b=a \cdot b$ (llueve y llevo paraguas) son adecuados. Sin embargo, el condicional material $a \rightarrow b=a^{\prime}+b$ (no llueve o llevo paraguas) lo es menos, al ocultar la relación entre llover y llevar paraguas.

La operación $a \rightarrow b=a^{\prime} \cdot b$ es un condicional, ya que $a \cdot\left(a^{\prime} \cdot b\right)=(a$. $\left.a^{\prime}\right) \cdot b=0 \cdot b=0<b$. Sin embargo, interpreta el enunciado condicional en la forma 'No llueve y llevo paraguas', que no es satisfactoria. De la misma forma, $a \rightarrow b=a^{\prime}+b$, si $a \cdot b=0$, o a.b, en otro caso, es 
un condicional ya que de $a \cdot(a \rightarrow b)=0$ en el primer caso, y $a \cdot(a \rightarrow$ $b)=a \cdot b$ en el segundo, resulta $a \cdot(a \rightarrow b) \leq b$. Como sea que, en las álgebras de Boole, $a \cdot b=0$ implica $b=b \cdot a+b \cdot a^{\prime}=0+a^{\prime} \cdot b=a^{\prime}$. $b, \rightarrow$ se simplifica así, $a \rightarrow b=b$, si $a . b=0$, o $a \cdot b$ en otro caso, $y$ facilita la interpretación:

- Si 'llueve' y 'llevo paraguas' son incompatibles, 'llevo paraguas'

- Si 'llueve' y 'llevo paraguas' no son incompatibles, 'llueve y llevo paraguas', que también es satisfactoria.

Es decir, al abordar la representación de un enunciado lingüístico condicional por una operación condicional $\rightarrow$, la misma debe interpretar correctamente el significado del enunciado en el contexto que corresponda.

En el caso de los sistemas borrosos basados en reglas, por ejemplo no siempre se dispone de conocimiento de las negaciones de los antecedentes al no corresponder las mismas con 'hechos'. En tal caso, no cabe representar las reglas por un condicional $a \rightarrow b$ en cuya expresión figure $a^{\prime}$.

\section{Los cuatro esquemas bajo el condicional material y $C_{\wedge}$}

Veamos con algún detenimiento el caso típico del cálculo proposicional clásico, es decir aquel en que se opera en una estructura de álgebra de Boole $\left(\mathrm{L}, \leq,+, \cdot,{ }^{\prime} ; 0,1\right)$. Recordemos, que el condicional material $a \rightarrow b$ se representa por $a^{\prime}+b$.

\section{1.}

$P=\{a \rightarrow b ; a\}, p_{\wedge}=a \cdot(a \rightarrow b)=a \cdot b$. Supóngase que es $a \cdot b \neq 0$.

- $C_{\wedge}(P)=\{q \in \hat{L} ; a \cdot b \leq q\}=\{q \in L ; q=a \cdot b+x ; \forall x \in L\}=a \cdot b+L$ Por ejemplo,

- Con $x=b, q=a \cdot b+b=b$ (Modus Ponens)

- Con $x=a, q=a \cdot b+a=a$

- Con $x=0, q=a \cdot b$

- Con $x=a+b, q=a+b$.

- $\operatorname{Conj}_{\lambda}(P)=\left\{x \in L ; a \cdot b \$ x^{\prime}\right\}$

Por ejemplo, si es $x^{\prime}<a \cdot b$, entonces $x \in \operatorname{Conj}_{\Lambda^{\prime}}(P)$

- $\operatorname{Ref}_{\wedge}(P)=\left\{x \in L ; a \cdot b \leq x^{\prime}\right\}=\left\{x \in L ; x \leq a^{\prime}+\hat{b}^{\prime}\right\}$.

Por ejemplo, $a^{\prime}+b^{\prime}, a^{\prime}, b^{\prime} \in \operatorname{Ref}_{\wedge}(P)$.

- $\operatorname{Hyp}_{\wedge}(P)=\{b \in L ; 0<b<a \cdot b\}$

- $\operatorname{Sp}_{\wedge}(\hat{P})=\left\{s \in L ; a \cdot b \ddagger s^{\prime} \& a \cdot b N C s\right\}$ 


\section{2.}

$P=\left\{a \rightarrow b, b^{\prime}\right\} p_{\wedge}=b^{\prime} \cdot(a \rightarrow b)=a^{\prime} \cdot b^{\prime}$. Supóngase que $a^{\prime} \cdot b^{\prime} \neq 0$ (o $a$ $+b \neq 1)$.

- $C_{\wedge}(P)=\left\{q \in L ; a^{\prime} \cdot b^{\prime} \leq q\right\}=a^{\prime} \cdot b^{\prime}+L$. Por ejemplo, $a^{\prime} \cdot b^{\prime}+a^{\prime}=a^{\prime} \in C_{\lambda}(P)$ (Modus Tollens)

- $\operatorname{Conj}_{\lambda}(P)=\left\{x \in L ; a^{\prime} \cdot b^{\prime} \ddagger x^{\prime}\right\}=\{x \in L ; x \supsetneqq a+b\}$. Por ejemplo, $a+b+x \in \operatorname{Conj}_{\wedge}(P)$.

- $\operatorname{Ref}_{\wedge}(P)=\left\{x \in L ; a^{\prime} \cdot b^{\prime} \leq x^{\prime}\right\}=\{x \in L ; x \leq a+b\}$

- $\operatorname{Hyp}_{\wedge}(P)=\left\{b \in L ; 0<b<a^{\prime} . b^{\prime}\right\}$. Por ejemplo, $b=a^{\prime} \cdot b^{\prime} \cdot x \in H_{y} p_{\lambda}(P) ; \forall x \in L$

- $S p_{\wedge}(P)=\left\{s \in L ; a^{\prime} \cdot b^{\prime} \ddagger s^{\prime} \& a^{\prime} \cdot \hat{b}^{\prime} N C s\right\}$

\section{3.}

$P=\{a \rightarrow b, b\}, p \wedge=b \cdot(a \rightarrow b)=b$. Supóngase que $b \neq 0$.

- $C_{\wedge}(P)=\{q \in L ; b \leq q\}=b+L$.

Por ejemplo, $b+a \in C_{\wedge}(P)$

- $\operatorname{Conj}_{\wedge}(P)=\left\{x \in L ; b \ddagger x^{\prime}\right\}=\left\{x \in L ; x \supsetneqq b^{\prime}\right\}$.

Por ejemplo, si $x^{\prime}<b$, entonces $x \in \operatorname{Conj}_{\wedge}(P)$.

- $\operatorname{Ref}_{\wedge}(P)=\left\{x \in L ; b \leq x^{\prime}\right\}=\left\{x \in L ; x \leq b^{\prime}\right\}$

- $\operatorname{Hyp}_{\wedge}(P)=\{b \in L ; 0<b<b\}$

- $S p_{\wedge}=\left\{s \in L ; b \ddagger s^{\prime} \& b N C s\right\}$

\section{4.}

$P=\left\{a \rightarrow b, a^{\prime}\right\}, p_{\wedge}=a^{\prime} \cdot(a \rightarrow b)=a^{\prime}$. Supóngase que $a^{\prime} \neq 0(a \neq 1)$.

- $C_{\wedge}=\left\{q \in L ; a^{\prime} \leq q\right\}=a^{\prime}+L$. Por ejemplo, $a^{\prime}+b \in C_{\wedge}(P)$.

- $\operatorname{Conj}_{\lambda}(P)=\left\{x \in L ; a^{\prime} \ddagger x^{\prime}\right\}=\{x \in L ; x \ddagger a\}$.

Por ejemplo, si $a<x$, entonces $x \in \operatorname{Conj}_{\wedge}(P)$ y, en particular $a+b \in$ $\operatorname{Conj}_{\lambda}(P)$.

- $\operatorname{Ref}_{\wedge}(P)=\left\{x \in L ; a^{\prime} \leq x^{\prime}\right\}=\{x \in L ; x \leq a\}$

- $\operatorname{Hyp}_{\lambda}(P)=\left\{b \in L ; 0<b<a^{\prime}\right\}$

- $S p_{\wedge}(P)=\left\{s \in L ; s \ddagger a \& s N C a^{\prime}\right\}$

\section{Nota}

1. Tanto el Modus Ponens, como el Modus Tollens clásicos, aparecen claramente en 3.1 y 3.2 y también se observa que en ambos casos se deducen otros elementos más que las correspondientes premisas.

En los esquemas 3.3 y 3.4, no sólo cabe deducir las segundas premisas $b$ y $a^{\prime}$, respectivamente, sino todos los elementos de la forma $b+L$ 
y $a^{\prime}+L$, en cada caso. Se tienen, además de la caracterización de las conjeturas, las de las hipótesis y las especulaciones. Así, en 3.4, si $s \in$ $L$ es tal que $a<s$ y $s \mathrm{NC}^{\prime}$, s es una especulación; si es $s=a+x \neq 1$, no puede ser $a+x \leq a^{\prime}$, que implica $a \cdot(a+x)=a=0$, pero puede ser $a^{\prime}$ $\leq a+x$, que implica $1=a+x$, luego $a+x \in S_{\wedge}(P)$.

2. En [8], Michael Dummett propone el esquema de razonamiento 'por casos':

$$
\begin{array}{r}
a \rightarrow b \\
a^{\prime} \rightarrow b \\
\hline b
\end{array}
$$

que en las álgebras de Boole es deductivo bajo $C \wedge$, ya que $(a \rightarrow b)$. $\left(a^{\prime} \rightarrow b\right)=\left(a^{\prime}+b\right) \cdot(a+b)=b$, con la única condición $b \neq 0$. Como $b$ $=a+a^{\prime}=1$, el esquema de Dummett permite deducir $a+a^{\prime}$ y $\left(a \cdot a^{\prime}\right)^{\prime}$ $=a+a^{\prime}$, pero al ser $b=a \cdot a^{\prime}=0$, no permite deducir $a \cdot a^{\prime}$.

\section{Los cuatro esquemas bajo el condicional material y $C_{s}$}

Para que se vea como, manteniendo el condicional material, los cuatro esquemas dependen estríctamente del sistema deductivo empleado, veámos brevemente que ocurre con ellos al considerar el operador de consecuencias $C_{\leq}$que es más débil que el $C_{\wedge}$, y que verifica $C_{\wedge}(P)=C_{\wedge}\left(\left\{p_{\wedge}\right\}\right)=C \leq\left(\left\{p_{\wedge}\right\}\right)$.

4.1.

$P=\{a \rightarrow b, a\}$.

- $C_{s}(P)=\left\{q \in L ; a^{\prime}+b \leq\right.$ ó $\left.a \leq q\right\}=\left\{a \in L ; a^{\prime}+b \leq q\right\} \cup\{q \in L ; a \leq q\}$. No se verifica el Modus Ponens, ya que de ser $a^{\prime}+b \leq b \Leftrightarrow a^{\prime}+b=b$, se tendría equivalentemente $a^{\prime} \leq b$, que no vale en general. De ser $a \leq$ $b$, de nuevo se encuentra una condición que no es general.

- $\operatorname{Conj}_{s}(P)=\left\{x \in L ; x^{\prime} \notin C_{s}(P)\right\}=\left\{x \in L ; a^{\prime}+b \neq x^{\prime}\right\} \cap\left\{x \in L ; a \leq x^{\prime}\right\}$

- $\operatorname{Ref}_{s}(P)=\left\{x \in L ; x^{\prime} \in C_{s}(P)\right\}=\left\{x \in L ; a^{\prime}+b \leq x^{\prime}\right\} \cup\left\{x \in L ; a \leq x^{\prime}\right\}$

\section{Nota}

Aunque $b$ no se deduce de $P$, hay muchos casos en los que es $b \in \operatorname{Conj}_{s}(P)$, es decir, $b$ es conjeturable dado $P$. En efecto, si fuese $b \in \operatorname{Ref}(P)$, bien sería $a^{\prime}+b \leq b^{\prime}$, bien $a \leq b$; en el primer caso seguiría $\left(a^{\prime}+b\right) \cdot b=b=0$ y en el segundo $a \cdot b^{\prime}=0$. Luego, si $b \neq 0$ y $a \cdot b^{\prime} \neq 0$, es $b \notin R e f_{s}(P)$ y por tanto $b$ $\in \operatorname{Conj}_{s}(P)$. En esas condiciones, $b$ es conjeturable de $P$. 
4.2.

$P=\left\{a \rightarrow b ; b^{\prime}\right\}$.

- $C_{s}(P)=\left\{q \in L ; a^{\prime}+b \leq q\right\} \cup\left\{q \in L ; b^{\prime} \leq q\right\}$.

No se verifica el Modus Tollens, ya que: $a^{\prime}+b \leq a^{\prime} \Rightarrow a^{\prime}+b=a^{\prime} \Leftrightarrow b$ $\leq a^{\prime}$, que no sucede en general. De ser $b^{\prime} \leq a^{\prime} \Leftrightarrow a \leq b$, tampoco sucede en general.

\section{3.}

$P=\{a \rightarrow b ; b\}$.

- $C_{s}(P)=\left\{q \in L ; a^{\prime}+b \leq q\right\} \cup\{a \in L ; b \leq q\}=\{a \in L ; b \leq q\}=b+L$, ya que $b \leq a^{\prime}+b$. En particular, $b, b+a, b+a^{\prime}$ se deducen de $P$.

4.4.

$P=\left\{a \rightarrow b ; a^{\prime}\right\}$

- $C_{s}(P)=\left\{q \in L ; a^{\prime}+b \leq q\right\} \cup\left\{q \in L ; a^{\prime} \leq q\right\}=\left\{q \in L ; a^{\prime} \leq q\right\}=a^{\prime}+L$, ya que $a^{\prime} \leq a^{\prime}+b$. En particular, $a^{\prime}, a^{\prime}+b, a^{\prime}+b^{\prime}$, se deducen de $P$.

\section{Notas 4.1}

1. En el esquema 4.2 y análogamente al 4.1, a' también puede conjeturarse de $P$. Es $\operatorname{Ref} \leq(P)=\left\{x \in L ; x^{\prime} \notin C_{s}(P)\right\}=\left\{x \in L ; a^{\prime}+b \leq x^{\prime}\right\} \cup\{x$ $\left.\in L ; b^{\prime} \leq x^{\prime}\right\}$, y si fuese $a^{\prime} \in \operatorname{Ref}_{s}(P)$ :

- $a^{\prime}+b \leq a^{\prime} \Rightarrow a^{\prime}+b=a^{\prime} \Leftrightarrow a \cdot b=0$, que no es el caso en general

- $b^{\prime} \leq a^{\prime} \Leftrightarrow a \leq b$, que tampoco es el caso en general.

Así, de no ser ni $a . b \neq 0$, ni $a \leq b$, cabe conjeturar $a^{\prime}$ en el esquema 4.2.

2. Por tanto, ni MP, ni MT son esquemas deductivos, pero si conjeturables.

3. A pesar de los fallos de MP y de MT, hay otros de los llamados 'esquemas básicos de deducción' que se mantienen. Por ejemplo,

- $p \cdot q: p$, ya que $P=\{p \cdot q\}, y p \cdot q \leq p$

- $p: p+q$, ya que $P=\{p\}, y p \leq p+q$

- $p \Delta q: p+q$, ya que $p \Delta q=(p+q) \cdot(p \cdot q)^{\prime}$ (diferencia simétrica), $y$ $p \Delta q \leq p+q$

- $p \Delta q: p^{\prime}+q^{\prime}$, ya que $p \Delta q=(p+q) \cdot\left(p^{\prime}+q^{\prime}\right), y p \Delta q \leq p^{\prime}+q^{\prime}$.

\section{Ejemplo: El juego de dados}

¿Qué tipo de razonamientos sobre el resultado obtenible hace alguien que juega a los dados? Para simplificar, supondremos que el jugador tira un único dado que no está trucado. 
La información disponible es que el resultado puede ser uno cualquiera de los números $1,2, \ldots, 6$ en cada tirada. Sobre tal información caben enunciados como los siguientes.

- Sacar un 6, que corresponde al subconjunto $\{6\} \subset\{1,2, \ldots, 5,6\}=X$

- Sacar un número impar: $\{1,3,5\} \subset \mathrm{X}$

- Sacar un número mayor que $3:\{4 ; 5 ; 6\} \subset X$.

Por tanto, el problema puede enmarcarse en el conjunto $P(\mathrm{X})$, de las partes de $\mathrm{X}=\{1,2, \ldots, 5,6\}$ y, por ejemplo:

- No sacar un 6: $\{6\}^{\mathrm{c}}=\{1,2, \ldots, 5\}$

- Sacar impar mayor que 3: $\{1,3,5\} \cap\{4,5,6\}=\{5\}$.

Es decir, el marco corresponde al álgebra de Boole $\left(P(X), \cap, \cup,{ }^{c}\right)$, y es razonable elegir el sistema deductivo dado por $C_{\Lambda}$, con $\mathfrak{F}=P(X)-\{\varnothing\}, P=$ $\{X\}, y p_{\wedge}=X$. Nótese que el jugador no va a apostar a 'no sacaré nada' (Ø), ni va a ser autorizado a apostar 'sacaré algún número' $(X)$; por tanto, los resultados significativos del juego son, realmente, los subconjuntos en $P(X)$ $-\{\varnothing, X\}$. Lo que es deducible es

con lo que:

$$
C_{\wedge}(\{X\})=\{Q \in P(X) ; X \subset Q\}=\{X\},
$$

$\operatorname{Conj}_{\wedge}(\{X\})=\left\{P \in P(X) ; X \not \subset X^{C}=\varnothing\right\}=\{P \in P(X) ; P \neq \varnothing\}=P(X)-\{\varnothing\}=\mathfrak{F}$,

$H y p_{\wedge}(\{X\})=\{P \in P(X) ; P \neq \varnothing \& P \neq X\}=P(X)-\{\varnothing, X\}$,

$S p_{\wedge}(\{X\})=\varnothing$.

Así, el jugador sólo puede deducir que sacará algún número entre 1 y 6 , y conjeturar las hipótesis en $P(X)-\{\varnothing, X\}$. Lo que puede deducir no le sirve para jugar; lo que le sirve son las hipótesis que puede conjeturar para apostar; el razonamiento deductivo es insuficiente para este tipo de problemas.

\section{Condicional e implicación}

Como se advierte en [10] y [13] para el caso del condicional material, los términos condicional e implicación no deben confundirse. Un condicional $\rightarrow$ es una operación del tipo $L \times L \rightarrow L$, en tanto que la correspondiente implicación $\Rightarrow$ es una relación binaria, es decir, un subconjunto de $L \times L$, que se obtiene al afirmar (absolutamente) el condicional:

$$
(a, b) \in \Rightarrow \text { o } a \Rightarrow b \text { ssi } a \rightarrow b=1 \text {. }
$$

Condicional e implicación son términos de naturaleza distinta, como en la Aritmética lo son la suma y la ordenación de los números, aunque ambos estén ligados por la definición: $\mathrm{r} \leq \mathrm{s}$ ssi existe un número $\mathrm{x}$ tal que $s=r+x$. 
Como se ha dicho, en el caso del condicional material es $a \rightarrow b=a^{\prime}+$ $b=1$ ssi $a \leq b$, es decir, que la implicación asociada $\Rightarrow$ es, exactamente, la relación de orden parcial propia del álgebra de Boole y, en el caso del condicional conjuntivo, es $a \rightarrow b=a \cdot b=1$ ssi $a=b=1$, con lo cual es $\Rightarrow=\{(1 ; 1)\}$.

Cada condicional $\rightarrow$ permite definir su bicondicional $a \leftrightarrow b=(a \rightarrow b) \cdot(b$ $\rightarrow a$ ), que también es un condicional con cuya afirmación absoluta se obtiene la relación de equivalencia lógica: $a \Leftrightarrow b$ ssi $a \leftrightarrow b=1$. En el caso del condicional material es $a \leftrightarrow b=\left(a^{\prime}+b\right) \cdot\left(b^{\prime}+a\right)=a \cdot b+a^{\prime} \cdot b^{\prime}$, que es igual a 1 si y sólo si es $a \leq b$ y $b \leq a$, es decir, $a \Leftrightarrow b$ ssi $a=b$ : la equivalencia lógica asociada es exactamente, en este caso, la igualdad propia del álgebra de Boole. En el caso del condicional conjuntivo, es $a \leftrightarrow b=a \cdot b, y \Leftrightarrow=\{(1 ; 1)\}:$ los enunciados lógicamente equivalentes son las tautologías. Con $a \rightarrow b=b$, es $\Rightarrow=\{(a ; 1)\}$, y también $\Leftrightarrow=\{(1 ; 1)\}$.

Con $a \rightarrow b=a^{\prime}$ es $\Rightarrow=\{(0 ; b)\}$ y $a \leftrightarrow b=a^{\prime} \cdot b^{\prime}=1$, o $a+b=0$, con lo que $\Leftrightarrow=\{(0 ; 0)\}$ : los enunciados equivalentes son las antilogías. Finalmente, con el condicional no expresable por conectivos

$$
a \rightarrow b=\left\{\begin{array}{l}
a^{\prime}+b, \text { si } a \cdot b=0 \\
a \cdot b, \text { en otro caso, }
\end{array}\right.
$$

es $\Rightarrow=\{(1,1)\} \cup\{(0, b)\}$, ya que de $a^{\prime}+b=1$ o $a \leq b$, sigue $a \cdot a \leq a \cdot b=0$, o $a=0$. En ese caso es

$$
a \leftrightarrow b=\left\{\begin{array}{l}
a^{\prime} \cdot b^{\prime}, \text { si } a \cdot b=0 \\
a \cdot b, \text { en otro caso, }
\end{array}\right.
$$

con lo que es $a \leftrightarrow b=1$ ssi cuando los enunciados son incompatibles son antilogías y cuando no, son tautologías.

En realidad el nombre de 'equivalencia lógica' de la relación binaria $\Leftrightarrow$ acabada de definir, sólo se aviene en el sentido algebraico usual al caso en que el condicional que permite definirla es el material $a \rightarrow b=a^{\prime}+b$. En él, al ser $a \Leftrightarrow b$ ssi $a=b$, es obvio que $\Leftrightarrow$ posee las propiedades reflexiva ( $a \Leftrightarrow$ $a$, para todo $a$ ), simétrica $(a \Leftrightarrow b$ ssi $b \Leftrightarrow a$ ), y transitiva (Si $a \Leftrightarrow b \& b \Leftrightarrow c$, entonces $a \Leftrightarrow c$ ); es decir, que $\Leftrightarrow$ es una relación algebraica de equivalencia. Óbviamente, sus clases de equivalencia constan de un único elemento: la clase de a sólo contiene al elemento a y, por tanto, el conjunto cociente $L / \Leftrightarrow$ es isomorfo al $L$, puede identificarse con $L$. Lo mismo ocurre con el caso del condicional $a \leftrightarrow b= \begin{cases}a^{\prime} \cdot b^{\prime}, & \text { si } a \cdot b=0 \\ a \cdot b, & \text { si } a \cdot b \neq 0 \text { : }\end{cases}$

Sin embargo, en otros casos como aquel en que $\Leftrightarrow$ viene definido por el condicional conjuntivo a $\rightarrow b=a \cdot b$ (o por el condicional $a \rightarrow b=a^{\prime}$ ), en el cual es $a \Leftrightarrow b$ ssi $a=b=1$ (respectivamente, sii $a=b=0$ ), se verifican las propiedades simétrica y transitiva, pero no la reflexiva ya que en el caso del condicional conjuntivo, y de forma análoga para el condicional $a \rightarrow b=a^{\prime}$, 
para que fuese $a \Leftrightarrow a$ para todo a, debería ser $a \cdot a=1$, es decir, $a=1$ : no habría más elemento que 1 y $L$ no sería un álgebra de Boole. Para obtener una equivalencia algebraica basta modificar la definición en la forma:

$$
a \Leftrightarrow^{*} b \text { ssi } a=b \text {, ó } a=b=1 \text { si } a \text { y } b \text { son distintos. }
$$

Que la implicación asociada al condicional material coincida exactamente con el orden parcial del álgebra de Boole, y la equivalencia con la igualdad, hace extraño que en el cálculo proposicional clásico no se consideren directamente tal orden y tal igualdad.

Además, los operadores $C_{\wedge}$ y $C_{\leq}$reproducen, por la vía ' $a \leq b$ ssi $b \in$ $C(\{a\})$ ', el orden parcial del álgebra de Boole:

$$
a \leq_{C_{A}} b \text { ssi } b \in C_{\wedge}(\{a\})=C_{\leq}(\{a\}) \text { ssi } a \leq b \text {; supuesto } a \neq 0 \text {. }
$$

En este sentido, tanto esos operadores como el condicional material, están bien ligados a la estructura de orden del álgebra de Boole. Otra razón que hace extraño que, en el cálculo proposicional clásico, no se tenga en cuenta el orden parcial del álgebra de Boole, una estructura que representa perfectamente el tal cálculo.

\section{Condicionales booleanos}

Si $\mathfrak{E}=\left(L, \leq, \cdot,+,{ }^{\prime} ; 0,1\right)$ es un álgebra de Boole, las funciones de dos variables de la forma

$$
f(a, b)=\alpha \cdot a \cdot b+\beta \cdot a^{\prime} \cdot b+\gamma \cdot a \cdot b^{\prime}+\delta \cdot a^{\prime} \cdot b^{\prime},
$$

con los coeficientes $\alpha, \beta, \gamma, \delta$ en $\{0 ; 1\} \subset L$ reciben el nombre de funciones booleanas.

Un condicional booleano, o material, es un condicional que puede escribirse en forma de una función booleana. De ellas hay ocho, que se encuentran como sigue.

De $a \rightarrow b=\alpha \cdot a \cdot b+\beta \cdot a^{\prime} \cdot b+\gamma \cdot a \cdot b^{\prime}+\delta \cdot a^{\prime} \cdot b^{\prime} \leq a^{\prime}+b=\left(a \cdot b^{\prime}\right)^{\prime}$, sigue $\gamma \cdot a \cdot b^{\prime}=0$ y $\gamma=0$; con ello

- $a \cdot(a \rightarrow b)=a \cdot\left(\alpha \cdot a \cdot b+\beta \cdot a^{\prime} \cdot b+\delta \cdot a^{\prime} \cdot b^{\prime}\right)=\alpha \cdot a \cdot b \leq b$

- $b^{\prime} \cdot(a \rightarrow b)=b^{\prime} \cdot\left(\alpha \cdot a \cdot b+\beta \cdot a^{\prime} \cdot b+\delta \cdot a^{\prime} \cdot b^{\prime}\right)=\delta \cdot a^{\prime} \cdot b^{\prime} \leq a^{\prime}$,

es decir, si $\alpha \neq 0$ y $\delta \neq 0$, las funciones booleanas $a \rightarrow b=\alpha \cdot a \cdot b+\beta \cdot a^{\prime} . b$ $+\gamma \cdot a \cdot b^{\prime}+\delta \cdot a^{\prime} \cdot b^{\prime}$ verifican tanto el Modus Ponens (MP), como el Modus Tollens (MT). Por lo tanto, se tiene la tabla: 


\begin{tabular}{|c|c|c|l|l|c|c|}
\hline No & $\alpha$ & $\beta$ & $\delta$ & $a \rightarrow b$ & MP & MT \\
\hline$(1)$ & 1 & 1 & 1 & $a \cdot b+a^{\prime} \cdot b+a^{\prime} \cdot b^{\prime}=b+a^{\prime} \cdot b^{\prime}=a^{\prime}+b$ & SI & SI \\
\hline$(2)$ & 1 & 1 & 0 & $a \cdot b+a^{\prime} \cdot b=b$ & SI & NO* \\
\hline$(3)$ & 1 & 0 & 0 & $a \cdot b$ & SI & NO $^{*}$ \\
\hline$(4)$ & 0 & 0 & 0 & 0 & NO* $^{*}$ & NO* \\
\hline$(5)$ & 0 & 0 & 1 & $a^{\prime} \cdot b^{\prime}$ & NO* $^{*}$ & SI \\
\hline$(6)$ & 0 & 1 & 1 & $a^{\prime} \cdot b+a^{\prime} \cdot b^{\prime}=a^{\prime}$ & NO* $^{*}$ & SI \\
\hline$(7)$ & 0 & 1 & 0 & $a^{\prime} \cdot b$ & NO $^{*}$ & NO* \\
\hline$(8)$ & 1 & 0 & 1 & $a \cdot b+a^{\prime} \cdot b^{\prime}=\left(a^{\prime}+b\right) \cdot\left(b^{\prime}+a\right)$ & SI & SI \\
\hline
\end{tabular}

Los casos en los que la verificación del MP o MT se marca con NO*, se debe a que nos encontramos ante situaciones del tipo $0 \leq b$ o $0 \leq a^{\prime}$, que no son en absoluto informativas ya que equivalen a $b \in L$ o $a^{\prime} \in L$, respectivamente. Por otra parte, se excluyó la posibilidad $p_{\wedge}=0$.

Nota 7.1 Las inecuaciones $a \cdot(a \rightarrow b) \leq \mathrm{b}(M P)$ y $b^{\prime} \cdot(a \rightarrow b) \leq a^{\prime}(M T)$, no se verifican únicamente para funciones booleanas $a \rightarrow b=\alpha \cdot a \cdot b+\beta \cdot$ $a^{\prime} \cdot b+\delta \cdot a^{\prime} \cdot b^{\prime}$. Por ejemplo, la antes citada

$$
a \rightarrow b= \begin{cases}a^{\prime}+b, & \text { si } a \cdot b=0 \\ a \cdot b, & \text { si } a \cdot b \neq 0,\end{cases}
$$

no es booleana ya que de serlo y si $a \cdot b=0$, sería $\beta \cdot a^{\prime} \cdot b+\delta \cdot a^{\prime} \cdot b^{\prime}=a^{\prime}+b$ y no hay ninguna combinación $(\beta, \delta)$ en $\{0 ; 1\}$ que la verifique:

- $\beta=1, \delta=1, a^{\prime} \cdot b+a^{\prime} \cdot b^{\prime}=a^{\prime}$

- $\beta=1, \delta=0, a^{\prime} \cdot b$

- $\beta=0, \delta=1, a^{\prime} \cdot b^{\prime}$

- $\beta=0, \delta=0,0$.

\section{Conclusiones}

\section{1.}

Para establecer el cálculo proposicional clásico es necesario no sólo disponer de un conjunto de elementos o fórmulas, sino además los conectivos con los que puedan formarse tales fórmulas, así como las que quepa deducir de ellas; es necesario, por tanto, establecerlo en una estructura algebráica $\mathfrak{E}=\left(L, \leq, \cdot,+,,^{\prime} ; 0,1\right)$, en la que $L$ representa los elementos a considerar, . representa la 'conjunción $y^{\prime},+$ la 'disyunción $o$ ', ' la 'negación no', 1 las tautologías y 0 las antilogías. Además, se precisa un operador $\rightarrow$ capaz de representar los enunciados condicionales Si/entonces. Tal cálculo no sólo 
presenta las propiedades típicas de los orto-retículos, sino que al ser $a \cdot b+$ $a \cdot b^{\prime}=a \cdot\left(b+b^{\prime}\right)=a \cdot 1=a$, por valer la ley distributiva, es necesariamente un álgebra de Boole de la que, usual y sorprendentemente, en el cálculo proposicional no se tiene en cuenta su orden parcial ' $a \leq b$ ssi $a \cdot b=a$ ssi $a+b=b$ ssi $a^{\prime}+b=1$ sii $b=a+a^{\prime} \cdot b^{\prime}$.

\section{2.}

Este breve artículo tiene por objetivo poner de manifiesto tres hechos. La indispensabilidad de encuadrar el cálculo proposicional clásico en el marco de las álgebras de Boole; la de emplear el mayor sistema deductivo posible en las álgebras de Boole, en el cual y aún oculto, se ha basado siempre el cálculo proposicional clásico y, finalmente, la de representar los enunciados condicionales mediante el llamado condicional material que, en las álgebras de Boole, no es sólo el mayor de los condicionales, sino, también, contrasimétrico y definidor del orden parcial $(p \leq q)$ cuando este se afirma como la tautología $p \rightarrow q=1$.

De pretender establecer el cálculo proposicional en estructuras algebraicas distintas de las booleanas, lo anterior deja de verificarse. Por ejemplo, tanto en los orto-retículos no booleanos, como en las álgebras de De Morgan no booleanas, el condicional material no verifica el Modus Ponens; además, no existe ningún condicional máximo sino, a lo más, maximal y que, además, no es contrasimétrico. Por ejemplo, en los retículos ortomodulares, el contrasimétrico del condicional (maximal) de Sasaki $a \rightarrow b=a^{\prime}+a \cdot b$, es el llamado de Dishkant $a \rightarrow b=b+a^{\prime} \cdot b^{\prime}$, que también es maximal [2].

Debe notarse que en las álgebras de Boole y gracias a las leyes distributivas, esos dos condicionales colapsan en el material: $a^{\prime}+a \cdot b=\left(a^{\prime}+a\right) \cdot\left(a^{\prime}\right.$ $+b)=1 \cdot\left(a^{\prime}+b\right)=a^{\prime}+b ; b+a^{\prime} \cdot b^{\prime}=\left(b+a^{\prime}\right) \cdot\left(b+b^{\prime}\right)=\left(b+a^{\prime}\right) \cdot 1=b+$ $a^{\prime}=a^{\prime}+b$. Análogamente, excepto en las álgebras de Boole, el operador $C \wedge$ no es el mayor posible y hasta pueden existir operadores de consecuencias no comparables con él.

Por todo ello, el marco natural del cálculo proposicional clásico es el álgebra de Boole. Un marco en el que, como se ha dicho en el apartado 2, son indistinguibles los conceptos de contradicción $\left(a \leq b^{\prime}\right.$ o $\left.a \rightarrow b^{\prime}=1\right) \mathrm{e}$ incompatibilidad $(a \cdot b=0)$.

\section{3.}

El cálculo proposicional booleano o clásico es propio para la deducción con enunciados precisos. Tan pronto se consideran, junto a estos enunciados, otros que no son precisos y bivaluados, el cálculo del que nos hemos ocupado solo rige para los segundos y deja de valer en cuanto una sola 
premisa no es precisa. Si, por ejemplo, se intenta deducir con enunciados precisos e imprecisos, hay que emplear la llamada lógica borrosa [18] en la que, por ejemplo, el Modus Ponens sólo vale en álgebras de conjuntos borrosos estríctamente dependientes de la particular representación que, en función del contexto, el uso de los términos en las premisas, y el propósito con el que se usen, quepa escoger para los enunciados condicionales [19]. Ningún álgebra de conjuntos borrosos es un álgebra de Boole, ni siquiera un orto-retículo y sólo existe un tipo de ellas que son retículos y, en ese caso, álgebras de De Morgan [12].

\section{Agradecimientos}

Este trabajo está parcialmente financiado por la Fundación para el progreso Soft Computing y el Ministerio de Educación y Ciencia (España), bajo el proyecto del CICYT TIN2011-29827-C02-01.

\section{Referencias}

[1] C. Vaucheret A. Fernandez Pineda, E. Trillas. Additional Comments on Conjectures, Hypotheses, and Consequences in Orthocomplemented Lattices. Fuzzy Logic in Knowledge-Based Systems, Decision and Control Eds, 107-114, 2001.

[2] L. Beran. Orthomodular Lattices. D. Reidel Pubs., 1985.

[3] G. Birkhoff. Lattice Theory. American Mathematical Society Colloquium Publications, 1967.

[4] G. Boole. The mathematical analysis of logic. Blackwell, London and Cambridge, 1847.

[5] G. Boole. The Laws of Thought. Macmillan, Londres, 1854.

[6] A. Deaño. Introducción a la lógica formal. Alianza Ed., Madrid, 1974.

[7] J. Delval and A. Rivière. Si llueve, Elisa lleva sombrero: Una investigación psicológica sobre la tabla de verdad del condicional. Revista de Psicología General y Aplicada., 30:825-850, 1975.

[8] M. Dummett. The Seas of Language. Oxford University Press, USA, 1996.

[9] E. Castiñeira E. Trillas, S. Cubillo. On Conjectures in Orthocomplemented Lattices. Artificial Intelligence, 117:255-275, 2000.

[10] J. Ferrater Mora, H. Leblanc. Lógica Matemática. Fondo de Cultura Económica, México, 1955. 
[11] M. Garrido. Lógica Simbólica. Tecnos, Madrid, 1973.

[12] A. Pradera, E. Trillas, and E. Renedo. An overview on the construction of fuzzy set theories. New Mathematics and Natural Computation, 1(3):329-358, 2005.

[13] W. Quine. El Sentido de la Nueva Lógica. Traducción castellana de Mario Bunge. Nueva Visión, Buenos Aires, 1958.

[14] W.V.O Quine. Los Métodos de la Lógica. Traducción castellana de M. Sacristán Luzón. Ariel, 1962.

[15] M.A. Quintanilla. Fundamentos de Lógica y Teoría de la Ciencia. Ediciones de la Universidad de Salamanca, 1981.

[16] H. Reichenbach. Elements of Symbolic Logic. Macmillan New York, 1947.

[17] J.F. Sowa. Knowledge Representation: Logical, Philosophical, and Computational Foundations. Brooks Cole Publishing, California, 2000.

[18] E. Trillas, C. Alsina, and J. M. Terricabras. Introducción a la Lógica Borrosa. Ariel, Barcelona, 1995.

[19] E. Trillas and I. García-Honrado. A reflection on the design of fuzzy conditionals. In "Experimentation and Theory - A Homage to Abe Mamdani” Springer-Verlang, 407-420, 2012.

[20] E. Trillas, I. García-Honrado, and A. Pradera. Consequences and Conjectures in Preordered Sets. Information Sciences, 180(19):3573-3588, 2010.

[21] E. Trillas, E. Renedo, and C. Alsina. On three laws typical of booleanity. In Proceedings NAFIPS '04, (2):520-523, Banf, Canada, 27-30 June 2004. 